Vierteljahrsschrift für Geschichte der Medizin und der Naturwissenschaften

Revue trimestrielle d'histoire de la médecine et des sciences naturelles Redaktion/Rédaction: Carl Haffter, Basel

\section{GESNERUS}

Jahrgang/Vol.39 1982

Heft/Fasc. 2

Verlag/Editions Sauerländer, Aarau

\title{
Die wiederaufgefundene «Pharmacia Contracta» des Johann Jakob Scheuchzer (1672-1733)
}

Von Jakob Büchi

\section{Einleitung}

Im Verzeichnis des wissenschaftlichen Nachlasses von Johann Jakob Scheuchzer, das Rudolf Steiger ${ }^{1}$, ehemaliger Bibliothekar an der Zentralbibliothek Zürich, in verdienstvoller Weise 1933 erstellt hat, sind zwei Handschriften medizinischen und pharmazeutischen Inhalts aufgeführt, die Scheuchzer im Privatunterricht von Medizinern benützte:

Hs.193: «Ludoviciana Pharmacia in compendium redacta». Zusammenfassung von D. Ludwig: «De pharmacia moderno saeculo accommodata» (Gotha 1671). Cit Dr. 70 (S.153). Wohl vor 1700. Vielleicht Vorstufe Hs. 194. Davon wahrscheinlich abhängig das Werk von Scheuchzers Schüler Wolfgang Christen «Thesaurus Ludovicianus sive compendium ... selectum ex Ludov. Pharmacia ...» (Berna 1707). Verschollen.

Hs. 194: «Pharmaciae ex variis autoribus contractae Clarior idea». Brit. Mus. Sloane Ms. 3455 (Bl.6-215). - Werk Scheuchzers von seinem Sohn Kaspar kopiert. Vermutlich Erweiterung von Hs. 193. Orig. verschollen.

Bei der Suche zürcherischer Handschriften und Druckwerke pharmazeutischen Inhalts fanden wir vor kurzem ein Exemplar der als verschollen 
bezeichneten Hs. 194 in der Bibliothek des Pharmazeutischen Institutes der Eidgenössischen Technischen Hochschule Zürich. Da das Werk einen interessanten Einblick in die Arzneitherapie und den Arzneischatz am Ende des 17.Jahrhundert erlaubt, soll es mit Bezug auf seine Vorlage, die «Pharmacia» Ludovicis, besprochen werden.

\section{Danielis Ludovicis «Pharmacia» $\mathbf{1 6 9 6}^{2}$}

Im Titelblatt zu seiner Handschrift (Abb. 3) vermerkt J. J. Scheuchzer ${ }^{3}$, daß er sich vornehmlich auf Ludovicus bezogen habe, weshalb dessen «Pharmacia» unsern Betrachtungen zugrunde zu legen ist; zudem drängen sich Vergleiche der beiden Werke auf.

\subsection{Der Autor}

Ludovicus, Daniel Ludwig (1625-1680), von Weimar, studierte an verschiedenen Universitäten, um 1647 in Jena zum Dr. med. zu promovieren. Nach Praxistätigkeiten in Jena und Königsberg (Franken) wurde er 1658 Stadtarzt in Salzungen und 1662 in Gotha. Ab 1666 wirkte er als Leibarzt und wurde Präsident des dortigen «Collegium medicum». Er war nicht nur als Arzt erfolgreich, sondern betätigte sich auch als wissenschaftlicher Schriftsteller. Er gab 1671 als epochemachendes Werk «De pharmacia moderno seculo accommodata» heraus, das verschiedene Auflagen erlebte und zur Vorlage der Hs. 194 von Scheuchzer ${ }^{3}$ wurde. Neben andern Schriften stammt von ihm auch das «Compendium materiae medicae», das allerdings erst 1698, d.h. nach seinem Tode, erschien.

\subsection{Aussehen und Einteilung der «Pharmacia»}

Von den verschiedenen Ausgaben (1671, 1685, 1688, 1693, 1696 und 1728) stand uns jene von 1696 (Lipsiae, Apud Joh. Herebordum Klosium) zur Verfügung und diente uns als Unterlage für vergleichende Überprüfungen. Dieses Buch von $10 \mathrm{~cm}$ Breite und $17 \mathrm{~cm}$ Höhe ist in Schweinsleder gebunden und gut erhalten. Es wird eingeleitet durch ein Porträt Ludovicis sowie der Darstellung eines Apothekerregals und von Bildchen einer Apotheke und eines Arbeitsraumes (Abb.1). Das Titelblatt (Abb.2) gibt darüber Auskunft, daß es sich beim vorliegenden Druck um die Editio Secunda von 1685 
mit einem Vorwort des berühmten D. Georg Wolfgang Wedelius und verschiedenen Ergänzungen handelt.

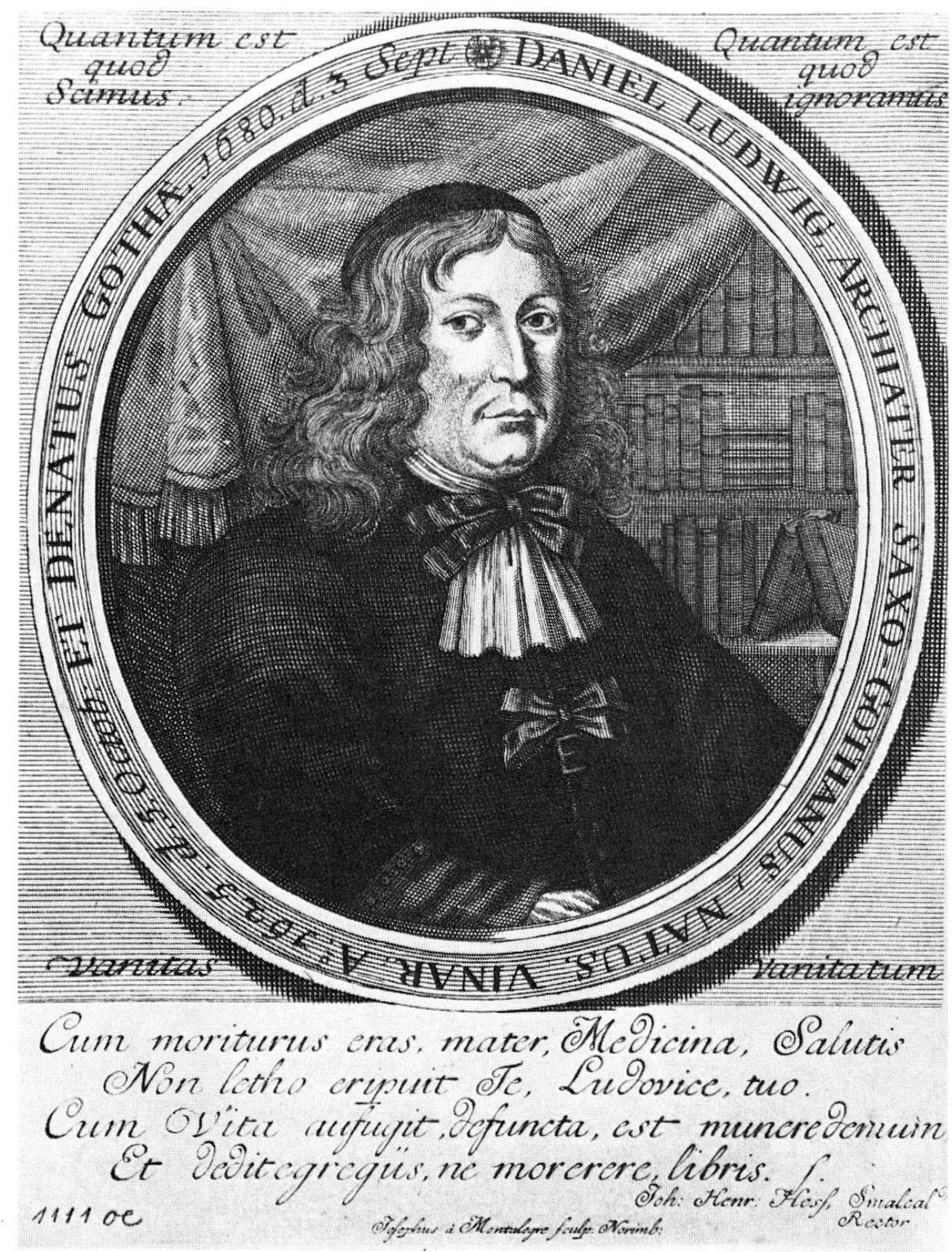

Abb. 1. Daniel Ludwig, Verfasser der «Pharmacia moderno Seculo accommodata» 
Als Einteilung wählte Ludovicus die folgende Disposition:

1. Praefatio: Lectore bene volo S.P.D., verfaßt von Georg Wolfgang Wedelius; XVS.

2. Praefatio ad Lectorem von Ludovicus; S. 1-9.

3. Introductio; S.10-21.

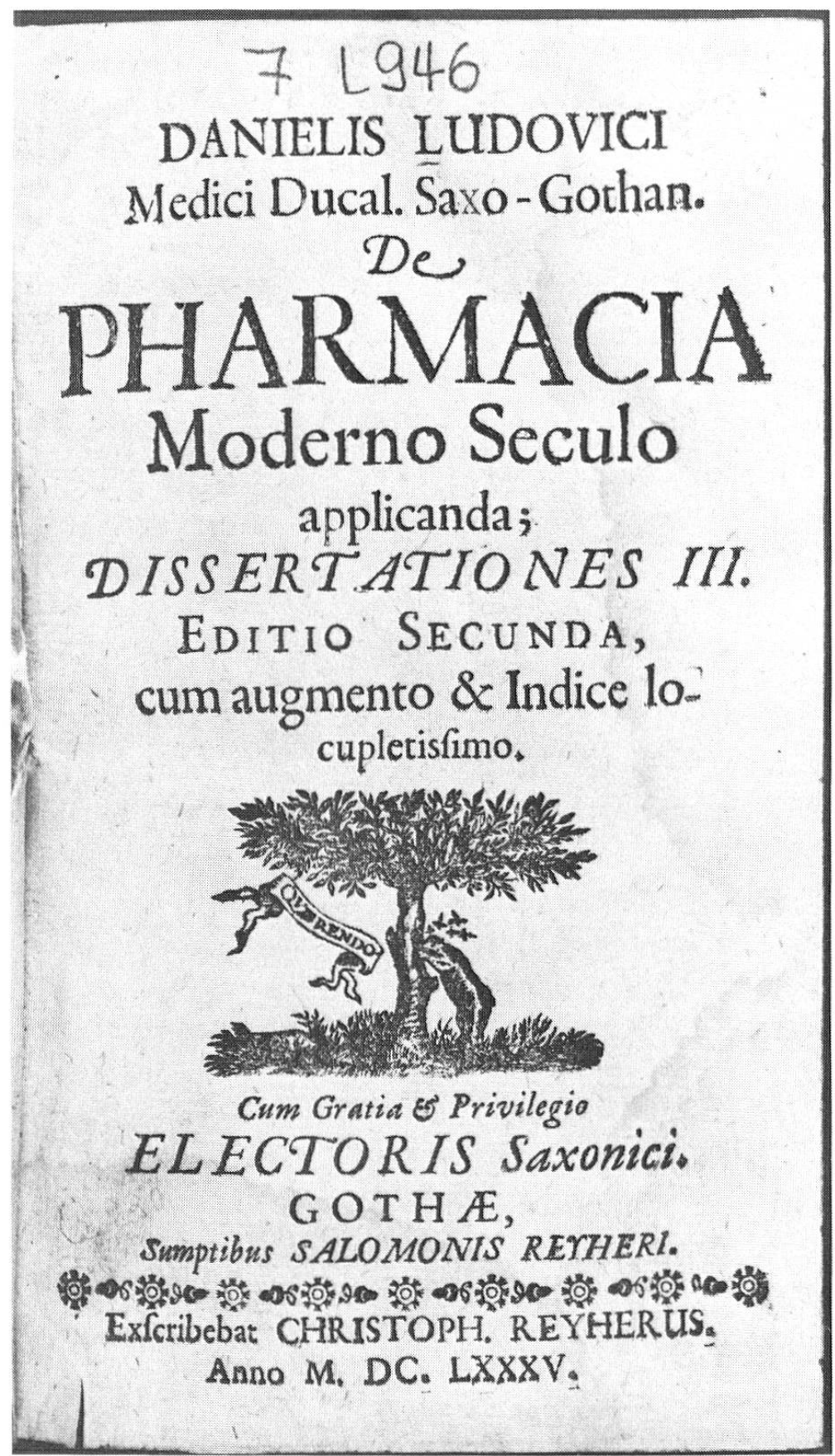

Abb.2. Titelblatt der «Pharmacia Moderno Seculo applicanda» 1685 des Daniel Ludovicus 
4. Dissertatio I: De Remediorum Selectu; S. 22-639.

In dieser Abhandlung bespricht der Autor die Anforderungen an die Arzneimittel und gibt einen Überblick über die Anwendung bei den verschiedenen therapeutischen Stoffklassen.

5. Dissertatio II: De Taxarum Moderatione; S. 640-697.

6. Dissertatio III: De Privata Remediorum Dispensatione: S. 698-749.

7. Ad praesationem: Ad Dissertationem I: S.1-93. Nachträge.

8. Index: $68 \mathrm{~S}$.

\subsection{Inhalt der «Pharmacia»}

Der Text der Dissertatio I ist nach therapeutischen Stoffklassen eingeteilt, von denen praktisch alle beschrieben sind, die gegen Ende des 17. Jahrhunderts gebraucht wurden (siehe Tab. I). Der Text bietet Angaben über die Wahl der Simplicia, mögliche und zu vermeidende Kombinationen derselben, die Beschreibung ihrer wichtigsten Präparate (Pulveres, Macerata, Infusa, Decocta, Essentiae, Tincturae und Extracta), sowie der verwendbaren Arzneiformen und der am häufigsten gebrauchten Composita. Dieser Teil der «Pharmacia» des Ludovicus lieferte vor allem Auszüge für die «Pharmacia Contracta» von Scheuchzer. Die Ausführungen enthalten viele kritische Bemerkungen über den Wert gewisser Arzneimittel und ließen Ludovicus zu einem Reformator der Pharmakotherapie und Pharmazie werden, indem er die Beseitigung einer Menge veralteter und unnützer Mittel empfahl. Diese kritische Beurteilung der Pharmakotherapie und der Pharmaka dürfte Scheuchzer ${ }^{9}$ veranlaßt haben, sich an Ludovicis «Pharmacia» anzulehnen.

\subsection{Die Materia Medica der «Pharmacia»}

Die Ausmittelung der behandelten Simplicia und Composita ergibt ein umfassendes Bild des gegen Ende des 17.Jahrhunderts bekannten Arzneischatzes. Insgesamt werden an die 1600 Arzneimittel aufgeführt, von denen 930 den Simplicia (410 Vegetabilia, 190 Animalia, 330 Mineralia und Chymica) sowie 670 den Composita zuzuteilen sind. Die Zahl der aufgeführten Pharmaka übertrifft vor allem dank der vielen Composita-Vorschriften jene der heute in Kraft stehenden Pharmakopöen. Unter den Vegetabilia finden wir praktisch alle aus dem Mittelalter und dem 16. Jahrhundert herübergenommenen pflanzlichen Arzneidrogen und Produkte; sie sind 
weitgehend ergänzt durch die nach der Entdeckung des Seeweges nach Ostindien in Europa eingeführten ausländischen Drogen, während die aus Amerika stammenden Arzneidrogen noch fast vollständig fehlen. Die große Zahl der durch Extraktion aus pflanzlichen Ausgangsstoffen hergestellten Arzneidrogenpräparate (2 Macerata, 6 Decocta, 6 Infusa, 8 Essentiae, 26 Extracta, 5 Magisteria und 20 Spiritus) läßt erkennen, daß das paracelsische Prinzip der Quinta essentia und ihrer Gewinnung sich bereits durchgesetzt hatte. Unerwartet umfangreich sind die Animalia, was dem Reformwillen von Ludovicus nicht ganz entspricht. Produkte von allen möglichen Tieren und vom Menschen (Lac, Pinguedo, Sanguis, Sevum und die verschiedensten Stercora) sind aufgeführt, das Aufkommen der «Dreckapotheke» Paullinis ${ }^{4}$ anzeigend. Bei den Mineralia und Chymica finden sehr viele, für die damalige Zeit neuartige chemische Substanzen Berücksichtigung, woraus ersichtlich ist, daß Ludovicus Anhänger der in der ersten Hälfte des 17. Jahrhunderts aufkommenden Chemiatrie war. Bei den Composita werden sehr viele Arzneipräparate hinsichtlich ihres pharmakotherapeutischen Wertes und ihrer Anwendung besprochen. Sie sind durch ihre Titel charakterisiert, welche auf die Arzneiform, die wirksamen Bestandteile und eventuell auf ihre Wirksamkeit oder Anwendung verweisen; Autoren von Vorschriften sind selten vermerkt. Hie und da gibt Ludovicus die qualitative Zusammensetzung ohne Mengenangaben, und nur selten werden Hinweise auf die Herstellung gegeben.

\subsection{Die «Pharmacia» des Ludovicus als Hilfsmittel der ärztlichen und pharmazeutischen Praxis}

Der große Wert dieses Werkes lag für die Ärzte vor allem in der pharmakotherapeutischen Wertung der Ausgangsstoffe für die Arzneipräparate und der Composita, während die Benützer für ihre Zubereitung auf weitere Literatur angewiesen waren. Dafür kamen die Arzneibücher jener Zeit in Betracht, vor allem die

«Pharmacopoea Colonienses» 1627;

«Pharmacopoea Dogmaticorum restituta» des Josephus Quercetanus, 1628; «Pharmacopoea Medico-pharmaceutica J.Schröderi», 1949;

«Pharmacopoea regia» des Johann Zwelffer, 1668;

«Pharmacopoea Augustana Reformata» 1675;

«Pharmacopoea Helveticorum» des J. Constant de Rebecque, 1677;

«Pharmacopée royale galénique et chimique» des Moysé Charas, 1676. 
Auch das von Ludovicus ${ }^{6}$ stammende «Compendium Materiae Medicae» 1698 kann als Unterlage für die fehlenden Angaben in der «Pharmacia» gelten.

Es ist verständlich, daß es die Ärzte wie z.B. Johann von Muralt ${ }^{5}$ mit seiner «Pharmacopoea Domestica» 1681 und J.J.Scheuchzer ${ }^{3}$ mit seiner «Pharmacia Contracta» als notwendig erachteten, eigene Arzneibücher zu schreiben, wo sie die Arzneimittel mit wichtigen Angaben über die Beschaffenheit der Ausgangsstoffe, die genaue Zusammensetzung und die Herstellung der Arzneipräparate versahen. Ähnliche Hilfswerke sind uns aus dieser Zeit auch von Apothekern überliefert; von H.C.Lavater ${ }^{7}$ stammt sein «Laborbuch» 1672 und von H.G. Lavater ${ }^{8}$ sind die «Collectanea Chymicopharmaceutica» 1674 erhalten.

\section{Johann Jakob Scheuchzers «Pharmacia Contracta» 1700}

Der berühmte Naturforscher und Arzt befaßte sich viele Jahre lang mit der Ausbildung von Medizinstudenten, die er im privaten Unterricht auf ihre Studien an den Universitäten vorbereitete. Auch diese Tätigkeit scheint mit ein Grund zur Abfassung der «Pharmacia Contracta» ${ }^{3}$ gewesen zu sein.

\subsection{Die Autoren}

Nach den Angaben des Titelblattes der «Pharmacia Contracta» (Abb. 3) hat M.D. Joh.Jakob Scheuchzer dieses Werk um 1700 verfaßt, während es von einem Med. Stud. Johann Scheuchzer geschrieben wurde. Wenn die Abschrift 1700 oder etwas später erfolgte, so handelt es sich bei diesem um den jüngern Bruder des Johann Jakob. Der Sohn Johann Kaspar Scheuchzer (1702-1729) kommt dafür kaum in Frage, da er erst ab 1725 Medizin studierte und 1728 den Doktorgrad erwarb.

Johann Jakob Scheuchzer, der Autor (1672-1733) ist als Naturforscher und Arzt vom Zürcher Medizinhistoriker Hans Fischer ${ }^{9}$ gebührend gewürdigt worden; seiner Beschreibung entnehmen wir den nachfolgenden Lebenslauf: Als Sohn des Stadtarztes Johann Jakob Scheuchzer (1645-1688), der sich neben seinem Arztberuf viel mit Anatomie und Botanik beschäftigte, erhielt er durch seinen Vater schon während seinen ersten Schuljahren in der Deutschen Schule, in der Lateinschule, im Collegium Humanitatis und am Carolinum viele Einblicke in die Naturwissenschaften. Zum Medizi- 
ner bestimmt, erhielt er schon vom 16. Lebensjahr an vorbereitenden medizinischen Unterricht durch seinen Vater und den Arzt Heinrich Lavater (1645-1719). Während seines Selbststudiums zur Vorbereitung auf die Universität begannen ihn Botanik und Mineralogie immer stärker zu beschäftigen. Mit einem Stipendium des Zürcher Rates konnte er die Universität Altdorf beziehen, wo er die erste medizinische Hochschulausbildung erhielt, sich aber auch für Botanik, Mathematik, Physik und Chemie

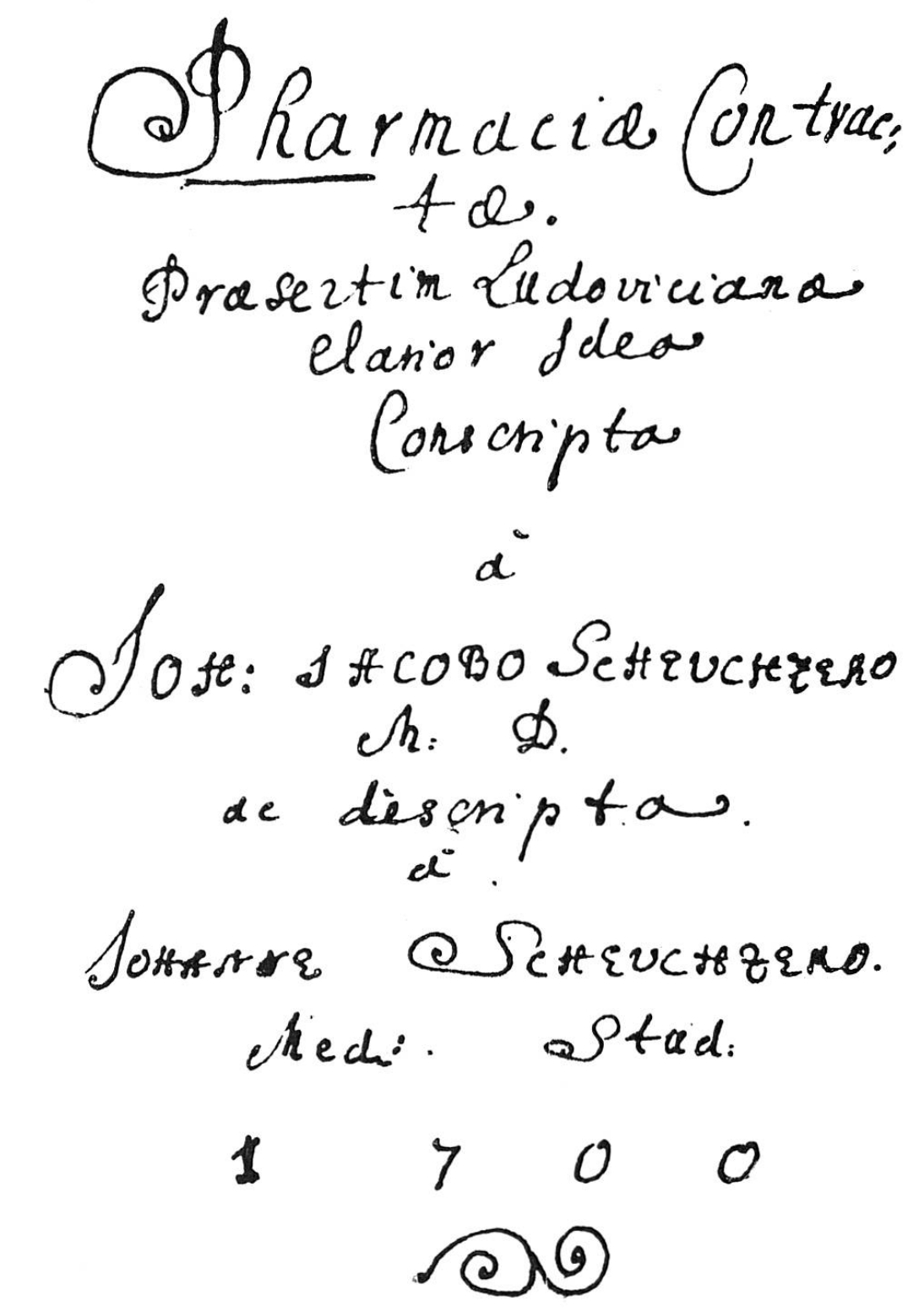

Abb. 3. Titelblatt der «Pharmacia Contracta» des Johann Jakob Scheuchzer 
interessierte. Die Beschäftigung mit Medizin trat bei Scheuchzer vorerst zurück. 1693 übersiedelte er an die Universität Utrecht, wo er sich während vier Monaten einem strengen Medizinstudium hingab. Dies führte 1694 zur Promotion zum Doctor medicinae. Seine Peregrinatio academica führte ihn über Belgien nach Süddeutschland zurück, wobei er vor allem botanische Gärten sowie mineralogische und zoologische Sammlungen besuchte. 1694 kehrte Scheuchzer nach Zürich zurück. Dort setzte er vorerst seine botanischen und steinkundlichen Forschungen fort und, da er über keine offizielle Stellung verfügte, eine halbakademische Tätigkeit begann, welche die Lücke zwischen Carolinum und Hochschule ausfüllte. Er hielt botanische und medizinische Vorlesungen und widmete sich vor allem der Vorbildung zukünftiger Medizinstudenten. Als Hilfsmittel zu diesem Zwecke stellte er unter andern seine «Pharamcia Contracta» zusammen, die zwischen 1695 und 1700 entstanden sein muß. Neben seiner ärztlichen Tätigkeit befaßte sich Scheuchzer mit mathematischen und naturwissenschaftlichen Studien paläontologischer und landeskundlicher Richtung, die ihn zum Universalgelehrten werden ließen. 1710 erhielt er die Professur für Mathematik am Carolinum, aber erst 1733 kam er zur längst verdienten Stelle des Physikprofessors und Chorherrn am Großmünster, die er von Johann von Muralt (1645-1733) nach dessen Ableben übernehmen konnte.

Johann Scheuchzer (1684-1738), der die Abschrift der «Pharmacia Contracta» besorgte, wurde von seinem ältern Bruder auf das Studium der Medizin vorbereitet, dem er in Basel oblag, wo er 1706 promovierte. Nach verschiedenartigen Tätigkeiten wurde er 1722 als Nachfolger seines Bruders zum Oberstadtarzt in Zürich sowie zum Professor der Physik und Chorherrn am Großmünster gewählt. Er machte sich vor allem verdient durch seine botanischen und geologischen Studien, verstarb aber schon früh, bald nach seinem Bruder.

\subsection{Aussehen und Einteilung der «Pharmacia Contracta»}

Das handschriftlich, von nur einer Hand stammende Werk ist ein gut erhaltener Pappband von $17 / 20 \mathrm{~cm}$ mit 251 halbspaltig beschriebenen Seiten. Die Einteilung des Buches entspricht jener, die damals üblich war für die Gruppierung der Arzneimittel und nach verschiedenen Gesichtspunkten (Herkunft, Form, Anwendung und Wirkung) vorgenommen wurde. Der Arzt Scheuchzer wählte als Haupteinteilung jene nach therapeutischen Stoffklassen, von denen berücksichtigt sind: 


\section{Tabelle I:}

Therapeutische Stoffklassen, aufgeführt in der «Pharmacia Contracta»

\begin{tabular}{ll}
\hline Adstringentia & Fumaria \\
Alexipharmaca (Gegengifte) & Hepatica \\
Alterantia (Säfte des Körpers/ & Hernearia \\
$\quad$ verändernde Mittel) & Hypnotica \\
Analeptica & Hypochondrica \\
Anodyna (schmerzstillende Mittel) & Laxantia \\
Antihysterica & Pectoralia \\
Antiscorbutica & Purgantia \\
Antivenerea & Refrigerantia (kühlende Mittel) \\
Aperientia & Resolventia (zerteilende Mittel) \\
Calida & Roborantia \\
Carminativa & Scabiosa \\
Cephalica (hauptstärkende Mittel) & Splenetica (milzwirksame Mittel) \\
Cordialia & Stomachica \\
Detergentia (vom Schleim & Temperata \\
$\quad$ reinigende Mittel) & Topica (örtlich wirkende Mittel) \\
Diaphoretica & Uterina \\
Diuretica & Vomitoria \\
$\quad$ befördernde Mittel) & Vulneraria \\
Epiceratica (die Schärfe der Säfte & \\
\hline
\end{tabular}

Die Anordnung dieser von Scheuchzer ${ }^{3}$ beschriebenen, längst nicht die Zahl der von W.Schneider ${ }^{10}$ für jene Zeit nachgewiesenen pharmakologischen Arzneimittelgruppen erreichend, erfolgte nicht alphabetisch; sie ist völlig willkürlich und läßt kein Ordnungsprinzip erkennen. Außerdem sind verschiedene Gruppen unterschiedlicher Wirkung und Anwendung (z.B. Aperientia, Detergentia, Hepatica und Uterina) in demselben Abschnitt zusammengefaßt. Die Übersichtlichkeit der Texte ist deshalb sehr gering und läßt sich nur durch Benützung des allerdings gut redigierten Index erreichen. Die verwendeten Gruppenbezeichnungen sind nur teilweise auf unsere Zeit übergegangen und haben zudem oft ihre Bedeutung geändert.

Die Zuteilung der einzelnen Arzneimittel zu den pharmakologischen Gruppen wurde nach der Wirkung, die effektiv vorhanden war oder an die man glaubte, vorgenommen. Da ihre Angriffspunkte kaum, die Wirkungsweise überhaupt noch nicht bekannt waren, kam es natürlich oft zu falschen 
Einteilungen, wie solche auch Scheuchzer ${ }^{3}$ unterliefen. Da Arzneimittel verschiedene Wirkungen besaßen oder ihnen solche zugesprochen wurden, treten zahlreiche der aufgeführten Pharmaka in verschiedenen therapeutischen Gruppen auf. Viele von ihnen galten als Panacea, Heilmittel, mit denen nach damaliger Ansicht den meisten oder allen Krankheiten beizukommen war.

\subsection{Die Quellen der «Pharmacia Contracta»}

Wie Scheuchzer ${ }^{3}$ im Titelblatt zu seinem Arzneibuch vermerkt, lehnte er sich vornehmlich Ludovicus ${ }^{2}$ an, aus dessen Schriften «De Pharmacia moderno saeculo accomodata» 1671, 1685, 1688 und 1698 er zahlreiche Angaben entnahm. Weitere Quellen sind in den Texten, meistens als Abschluß einer Simplicia-Beschreibung oder in einer Composita-Vorschrift vermerkt. Häufig sind die Hinweise auf die Arzneibücher des Quercetanus, Schröderus und Zwelfferus (siehe vorn, 1.5). In zahlreichen CompositaVorschriften sind berühmte Ärzte als Autoren aufgeführt. Interessanterweise wird nirgends auf die Schriften der Zürcher Conrad Gessner ${ }^{11}$, Caspar Wolf $^{12}$, Adrian Ziegler ${ }^{13}$ und Johann von Muralt ${ }^{5,14}$ Bezug genommen.

\subsection{Darstellung der Texte und Beschreibung der Arzneimittel}

Den Titeln der Arzneimittelgruppen, denen Scheuchzer ${ }^{3}$ keine Definitionen und therapeutische Hinweise aus Ludovicus ${ }^{2}$ anschloß, folgt jeweils die Beschreibung der Pharmaka. Wie das Beispiel der Purgantia zeigt, werden in der Regel zuerst die Simplicia, dann die Composita und schließlich die Rezept-Formeln angegeben:

«Purgantia vegetabilia:

Simplicia:

Julapa (Radix, Pulvis, Magisterium);

Aloe (Succus, Pilulae Aloeticae);

Senna (Folium, Pulvis, Infusum);

Scammonium (Succus, Pulvis, Magisterium seu Rosina,

Tinctura Rubinea solutiva Zapat., Diagridium);

Rhabarbarum (Radix, Tinctura, Anima Rharbari Wedelii) usf.

Purgantium Formulae (Pulveres, Pilulae, Morsuli, Electuaria,

Potiones, Emulsiones, Infusa);

Laxantia Formulae (Pulveres, Infusa, Potiuncula, Trochisci, Passulae).» 
Bei allen Arzneimittelgruppen werden somit zuerst die Simplicia (Vegetabilia, Animalia und Mineralia) hinsichtlich Stammpflanze, Herkunft, wenn nötig Zubereitung, Aussehen und Eigenschaften beschrieben. Dann wird einläßlich Auskunft gegeben über die wichtigsten Anwendungen, Kombinationen mit andern Arzneistoffen und selbst mögliche Nebenwirkungen (siehe Abb. 4, Aloe-Vorschrift).

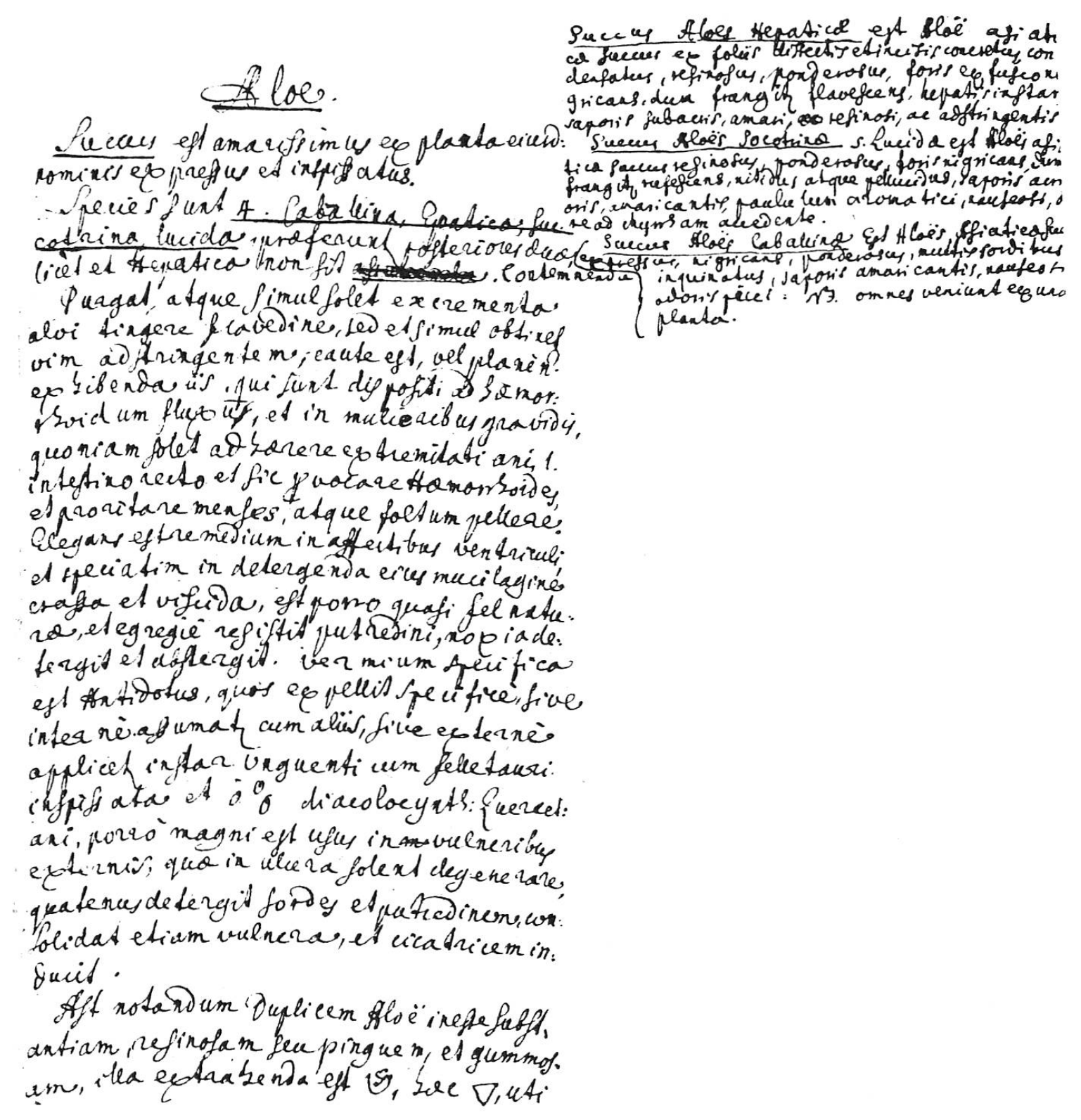

Abb.4. Text über die Aloe aus der «Pharmacia Contracta» des Johann Jakob Scheuchzer 
Es folgen Anweisungen für die Verarbeitung der Simplicia zu Arzneipräparaten wie Infusa, Decocta, Extractiones usf.). Hierauf schließen die wichtigsten Vorschriften für die Compositae und Formulae an. Diese sind zum Teil den gebräuchlichen Pharmakopöen und privaten Arzneibüchern entnommen. Einige davon sind von berühmten Ärzten überliefert (Abb.5):

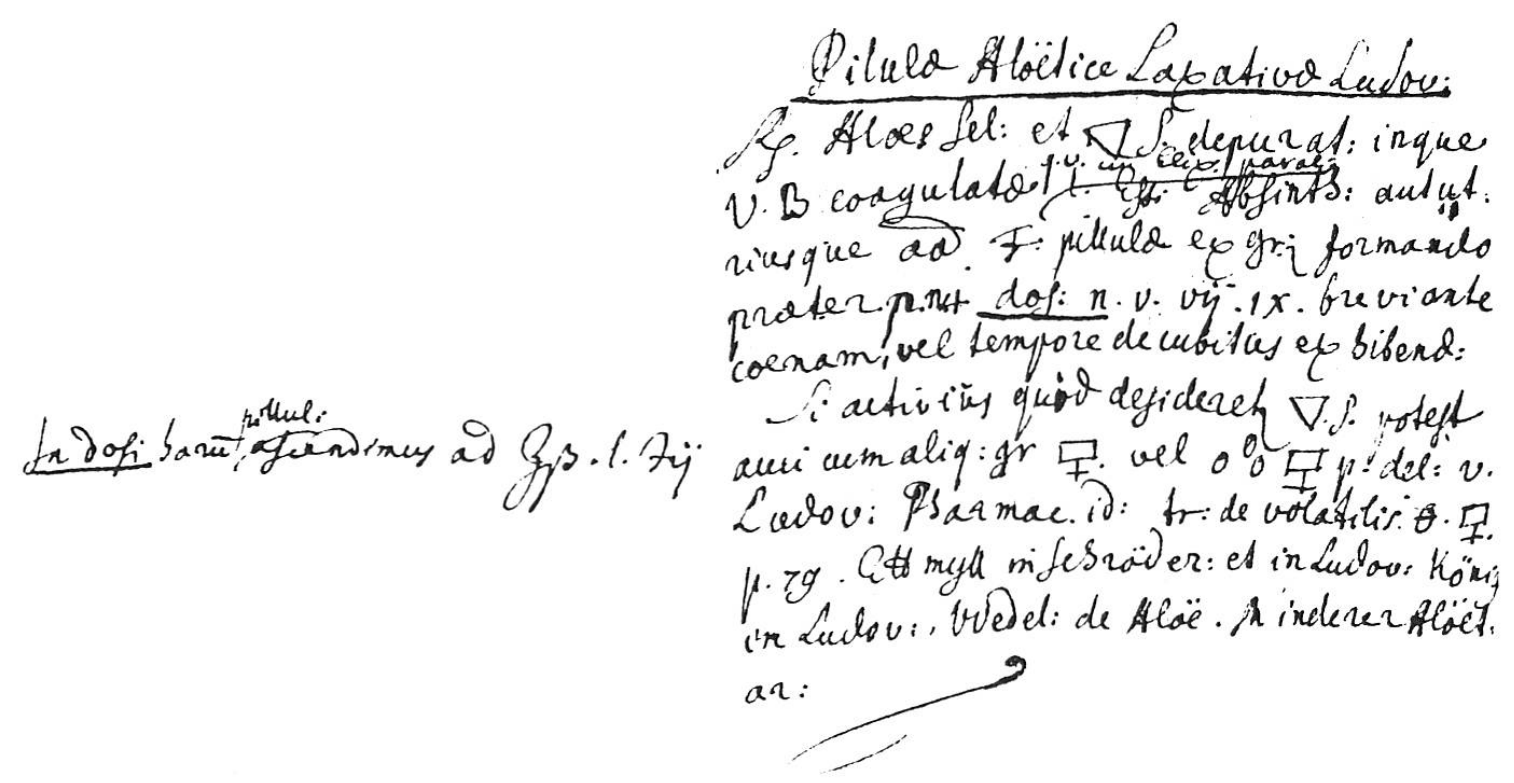

Abb.5. Vorschrift für Pilulae Aloeticae Laxativae Ludovici aus der «Pharmacia Contracta» des Johann Jakob Scheuchzer

Am Schluß der Texte der einzelnen therapeutischen Gruppen fügte Scheuchzer ${ }^{3}$ verschiedene einfache, aus seiner Praxis stammende Rezeptvorschriften an. Sie sind nicht mit Titeln versehen wie die als Composita bezeichneten Arzneipräparate (siehe Abb.6).

Diese Hinweise auf Composita- und Rezeptur-Vorschriften zeigen, daß Scheuchzer ${ }^{3}$ seine «Pharmacia Contracta» nicht nur als Auszug aus den pharmakotherapeutischen Beschreibungen von Ludovicus ${ }^{2}$ gestaltet hat, sondern die von diesem vorgeschlagenen Simplicia und Composita durch exakte Vorschriften über deren Zusammensetzung und Zubereitung ergänzte. Auf diese Weise stellte er seinen Schülern und Arztkollegen ein sehr brauchbares Arzneiverordnungsbuch zur Verfügung. 
Chagfe Catsolicepresty axy.

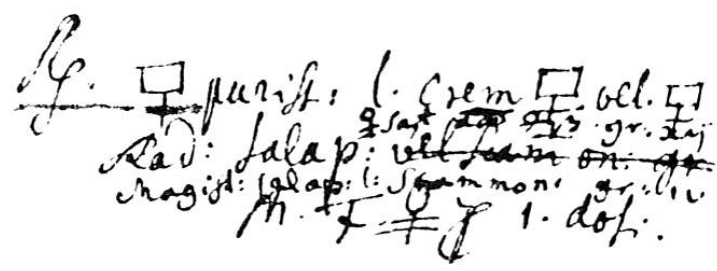

$$
\begin{aligned}
& \text { fo pulv: falap: } 7 v . \\
& \text { of nucills eqn: } 7
\end{aligned}
$$

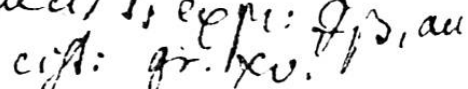

$$
\begin{aligned}
& \text { M. F. q o s. wel b. dofibe }
\end{aligned}
$$

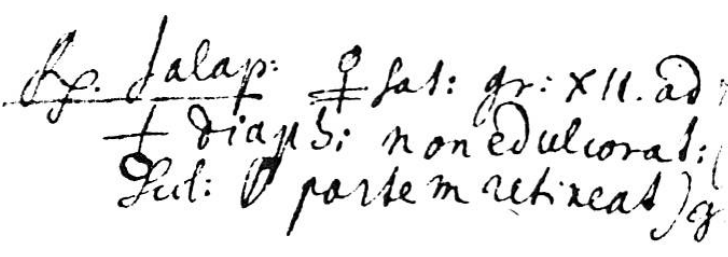
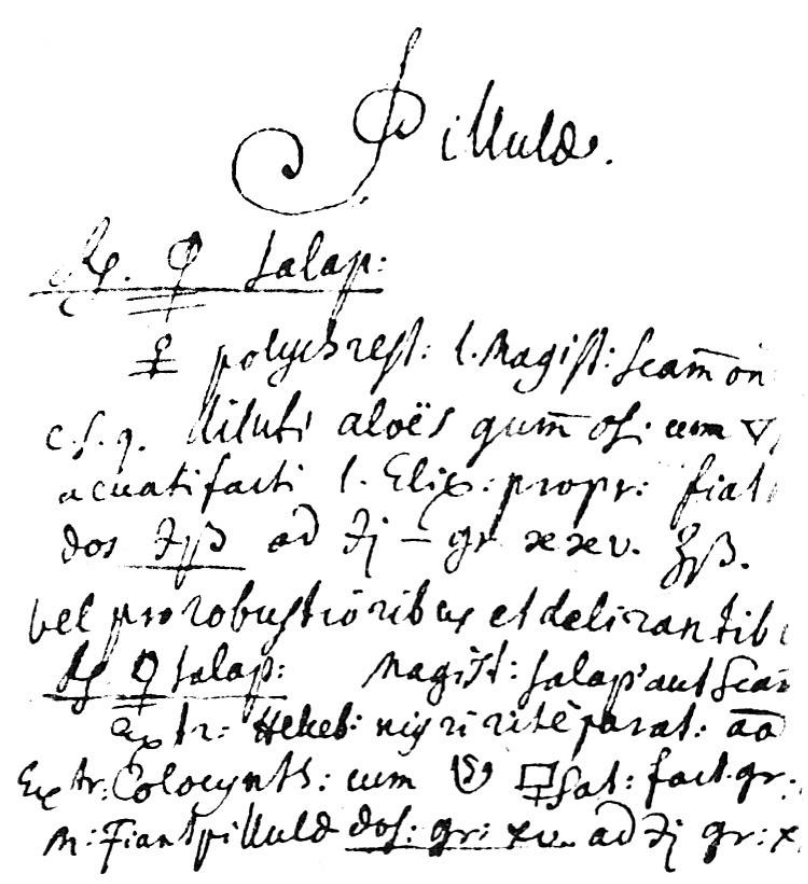

Abb.6. Rezept-Beispiele für die Purgantia in der «Pharmacia Contracta» des Johann Jakob Scheuchzer 


\subsection{Die Materia Medica der «Pharmacia Contracta»}

\subsubsection{Allgemeines}

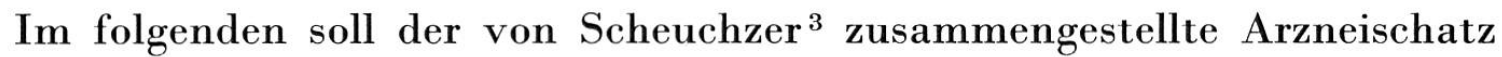
darauf untersucht werden, wie weit er den von Ludovicus ${ }^{2}$ beschriebenen übernahm und dem am Ende des 17. Jahrhunderts gebräuchlichen entsprach. Dabei interessierte auch festzustellen, ob dabei die ostasiatischen und amerikanischen Drogen berücksichtigt, sowie die mittelalterlichen tierischen Arzneistoffe beibehalten wurden und ob die Fortschritte der Chemiatrie zum Ausdruck kamen.

Scheuchzer ${ }^{3}$ hat den Arzneischatz seiner «Pharmacia Contracta» ganz erheblich reduziert gegenüber dem von Ludovicus ${ }^{2}$ vorgeschlagenen, und zwar auf etwa einen Drittel:

\begin{tabular}{lcccc}
\hline & Ludovicus & \multicolumn{2}{c}{ Scheuchzer } \\
\hline Simplicia & & 930 & & 268 \\
$\quad$ Vegetabilia & 410 & & 137 & \\
$\quad$ Animalia & 190 & & 42 & \\
$\quad$ Mineralia & 330 & 670 & 89 & 256 \\
Composita & & 1600 & & 524 \\
Total & & & & \\
\hline
\end{tabular}

\subsubsection{Die Simplicia}

Bei den Simplicia überwiegen die Vegetabilia noch ganz erheblich; sie umfassen einen großen Anteil von Arzneipflanzen, die heute nicht mehr offizinell sind. Dagegen sind die aus Ostasien neu eingeführten Drogen nur teilweise vertreten, und zwar mit Aloe, Benzoe, Camphora, Cassia, Cubeba, Opium, Moschus, Moscata, Myrrha, Rhabarbarum, Santalum und Zedoaria. Amerikanische Drogen dagegen sind kaum berücksichtigt; es sind nur Balsamum peruvianum, Jalapa, Lignum Guajaci, Nicotiana tabacum und Radix Sarsaparillae beschrieben. Vor allem fehlen Cortex Cinchonae, Folium Cocae und Radix Ipecacuanhae. An Zubereitungen von Vegetabilia finden sich in der «Pharmacia Contracta» schon zahlreiche Elixiria, Essentiae, Extracta und Tincturae, woraus zu schließen ist, daß die Idee der Quinta essentia des Paracelsus und ihre Verwirklichung durch Extraktion von Arzneidrogen eine schon weitgehende Realisierung gefunden hatte. 
Überraschend groß ist die Zahl der Animalia, die von Scheuchzer ${ }^{3}$ übernommen wurden. Von den meisten Tieren, die in frühern Jahrhunderten Arzneistoffe lieferten, verwendete er weiterhin die ganzen Tiere oder Teile davon (Cantharides, Lumbrici, Scorpiones und Vipera; Conchae, Cornu Cervi, Oculi Cancrorum und Unicornum), deren Sekrete und Ausscheidungen (Ambra, Fel Tauri, Moschus und verschiedene Stercora), auch das Blut der verschiedensten Tiere sowie deren Butter, Fette und Wachse. An menschlichen Produkten ist lediglich Harn vermerkt, der zu verschiedenen Spiritus-Präparaten verarbeitet wird. Der alte Wunderglaube an viele dieser tierischen Produkte und ihre Präparate war längst noch nicht überwunden. Von den Mitteln der «Heylsamen Dreckapotheke» 1696 des Franz Paullini ${ }^{4}$, die versprach, mit Kot und Harn fast alle Krankheiten zu heilen, war Scheuchzer ${ }^{3}$ noch kaum beeinflußt. Von diesen vielen Animalia sind heute nur noch wenige offizinell, so die Cantharides (Cantharidin), Adeps Suillus, Cera, Fel Tauri, Gelatina, Mel und Sperma Ceti.

Auch die Mineralia und Chymica sind auffallend gut vertreten; wie ein Vergleich mit den nach den Untersuchungen von W.Schneider ${ }^{10}$ während den Perioden der Vorchemiatrie (1500-1600), der Chemiatrie (1600-1670) und der Nachchemiatrie (nach 1670) neu eingeführten chemischen Arzneistoffe zeigt, sind die meisten davon von Scheuchzer ${ }^{3}$ aufgenommen worden. Wir finden vor allem die Stoffgruppen der Aquae destillatae plantarum, die anorganischen Säuren (Spiritus), Magisteria, weitere Metallsalze und die Olea destillata chimica. An organischen Arzneistoffen sind erst wenige (Camphora, Flos Benzoes, Pertoleum, Saccharum und Spiritus Vini) vermerkt.

\subsubsection{Die Composita}

An Composita sind ebenfalls zahlreiche Beispiele für applikationsbereite Arzneipräparate in der «Pharmacia Contracta» beschrieben. Sie sind durch bestimmte Titel gekennzeichnet, die von früher übernommen wurden. Diese umfassen Hinweise auf die Arzneiform, die Ausgangsstoffe oder auf Bestandteile, die Herstellung, die Wirkung oder Anwendung; zum Teil auch die Angabe des Autors der Vorschrift; also z. B.

- Tinctura Rhabarbari (aus Rhabarberwurzel hergestellt),

- Emplastrum Saturninum (bleihaltig),

- Oleum Hyoscyami expressum (ausgepreßtes Öl),

- Unguentum hypnoticum anodynum (schmerzstillend),

- Pilulae Nephreticae Wepferi (von Wepfer stammende Vorschrift). 
Interessante Vorschriften gehen auf berühmte Ärzte zurück; von den wichtigsten erwähnen wir einige Präparate:

Boe de le, Franz, genannt Sylvius (1614-1672); Arzt und Professor der Medizin in Leyden; galt als Haupt der Chemiatriker; Anatom, Physiologe und Kliniker; Förderer der Medizin:

- Sal Volatile Oleosum Sylvii und

- Sal Volatile Stomachicum Sylvii.

Ettmüller, Michael (1644-1683); Dr. med. 1668, Professor der Chirurgie an der Universität Leipzig, große praktische Fähigkeiten, tiefgründiger Gelehrter und eifriger Vertreter der Chemiatrie:

- Balsamum Vulnerarium Ettmülleri und

- Emplastrum ad menthem Ettmülleri.

Fabry, Wilhelm, genannt Fabricius Hildanus (1560-1634); berühmter Wundarzt, der auch in andern medizinischen Disziplinen gut ausgebildet war. Tätig in Köln, Lausanne, Payerne und ab 1615 Stadtarzt in Bern. Verfaßte zahlreiche medizinische Werke, vor allem die «Opera quae sunt omnia», Frankfurt 1646:

- Diaphoreticum mercuriale Fabry.

Hohenheim von, Theophrastus, genannt Paracelsus (1493-1541); kurze Zeit Stadtarzt in Basel, dann unsteter Wanderarzt. Erneuerer der Medizin und Begründer der Chemiatrie:

- Elixir Proprietatis Paracelsi.

Ludwig, Daniel, genannt Ludovicus (1625-1680?); Dr. med. Stadtarzt, Verfasser verschiedener wissenschaftlicher Schriften (siehe vorn, 1.):

- Aqua Cephalica externa Ludovici,

- Liquidum aloeticum Ludovici,

- Pulvis Antepilepticus Ludovici,

- Pulvis Stypticus Ludovici

und weitere 13 Vorschriften.

Langelott, Joël (1617-1680); Dr. med. 1647; Leibarzt und Leiter eines chemischen Laboratoriums; Feldarzt in vielen Kriegszügen; verfaßte eine Dissertation «De dolore dentium»:

- Extractum Antihypochondricum Langelottii.

Michaelis, Johann (1606-1667); Dr. med. 1631 in Leipzig, Professor für Pathologie und Therapie, dann Professor für Medizin und Therapie. Ab 1641 
Leibarzt des Kurfürsten von Sachsen. Bedeutender Chemiker und Anhänger der Chemiatrie:

- Pulvis Pectoralis Michaeli.

Ruland, Martin, genannt Rulandus (1532-1602); berühmter Arzt, wurde Leibarzt des Pfalzgrafen Philipp Ludwig, Anhänger des Paracelsus; verfaßte verschiedene medizinische Schriften:

- Emplastrum Diasulphuris Rulandi.

Screta, Heinrich (1637-1689); wirkte als Stadtarzt von Schaffhausen; bekannt als Verfasser der Schrift «Über die Lagersucht», in welcher er den Arzneischatz seiner Zeit kritisch besprach:

- Elixir Antihystericum Screta,

- Emplastrum Rubeum sticticum Screta,

- Sacculus Stomachicus Screta,

- Species pro Sacculo Stomachico Screta und

- Trochisci Hermetici Screta.

Wedel, Georg Wolfgang, genannt Wedelius (1645-1712); Studium der Medizin in Jena, praktische Tätigkeit, schließlich in Jena, dann 1667 Stadtarzt in Gotha; 1672 Promotion in Jena; Lehrstuhl der Medizin. Fürstlicher sächsischer Leibarzt. Berühmter Universitätslehrer, der zahlreiche Schriften hinterließ, die auch Pharmazeutisches behandeln:

- Anima Rhabarbari Wedelii,

- Linimentum Cordiale Wedelii,

- Oleum Polychrestum Wedelii,

- Spiritus Bezoardicus simplex Wedelii,

- und weitere acht Composita-Vorschriften.

Wepfer, Johann Jakob (1620-1695); Stadtarzt in Schaffhausen; stellte früh systematische toxikologische Untersuchungen an Tieren an und veröffentlichte zahlreiche wissenschaftliche Beobachtungen:

- Pilulae Nephreticae Wepferi und

- Pulvis stypticus Wepferi.

Würtz, Felix (Wirtz) (ca. 1500-ca. 1595); von Zürich, Lehre als Barbier, Reisen als Geselle, 1536-1559 Praxis als Scherer in Zürich; berühmter Wundarzt von 1559 bis zum Tode in Straßburg. Bedeutendstes Werk sind die «Practica der Wundarztney», Basel 1563, davon zahlreiche Auflagen:

- Unguentum fuscum Würtzii. 
Zapata, Giovanni (1520-?); promovierte als Dr. med. in Rom, verfolgte als praktischer Arzt in Rom eine einfache Heiltherapie vor allem mit Pflanzendekokten. Schrift über die Zubereitung von Arzneien «Maravigliosi secreti di medicina e cerugia», Rom 1586:

- Aqua Rubinea solutiva Zapati.

Mit diesen Composita-Vorschriften von ärztlichen Autoritäten des 16. und 17. Jahrhunderts hat Scheuchzer ${ }^{3}$ bekannte und viel gebrauchte, heute allerdings überholte Arzneimittel der wichtigsten therapeutischen Gruppen festgehalten.

\subsection{Beschreibung der Composita- und Rezept-Vorschriften}

Im folgenden soll etwas näher auf die Schreibweise und den Inhalt der verschiedenen Vorschriften eingegangen werden. Ihre Zusammensetzung, Herstellung und Anwendung sei anhand des ältesten aufgeführten Beispiels derselben, am «Elixir Proprietatis Paracelsi», besprochen. Diese Vorschrift hat Paracelsus im Archidoxis, Liber octavus «De elixiriis» etwa 1530 niedergelegt; sie ist von Karl Sudhoff ${ }^{16}$ wie folgt reproduziert worden:

Das ped felifir proprictatio.

2llfo audb bic natur cin clipir geboren bat, das vou rebus natmalibus mag in fein cigen wefen ein volfomen clipir gemad)t werben, als ates myrrba, fafrad) tmo alocpatico citrino. alts whs fraft inen cin folds Eompt, fezen wir be portm generatione, who figniren bie an oen procef uns laffen alfo ans feinen anfang, ben wir san wor side gensei baben, amo ift alfo.

Zie. myubat, aloepatici, croci ana l vierling; lafi in peli. canto mit arena afendiren anf bas miltept, steen monst. oarmad) feparit pex alembicum beriber bas olem vou faccibus fitue abuftione uno bas oleum bigerir mit circulato ein monat in gleidem gewdid, barmad) bebaltg. in bem if alle tugent ocr natïrliden balfamen wno ift in im virtus confervativa in fenibus, quos licitum non eft alfignanbo. quia non unans aetatem percurrit ab ea, fed quatuor, fed fepten, feo becem, chitug vim naturae polfe non eft pennae pro alligunnoo, etiam irriforium confultis boe legere, fed quo as noftrum ingenitum nobis fat eft interpretatum.

\author{
Abb. 7. Das \\ Originalrezept \\ für das \\ Elixir \\ Proprietatis \\ Paracelsi \\ aus dem \\ Archidoxis \\ Liber \\ octavus
}


Die Vorschrift wurde 1633 von Oswald Croll ${ }^{17}$ in seine «Basilica Chymica» und 1649 in die "Pharmacopoea Medico-Chymica» des Johann Schröder ${ }^{18}$ übernommen. Die genau abgefaßte Vorschrift von Croll ${ }^{17}$ lautet (siehe Abb.8).

Scheuchzer ${ }^{3}$ endlich gibt eine von Ludovicus ${ }^{2}$ modifizierte Vorschrift wieder (siehe Abb.9).

Ein Vergleich der obigen drei Vorschriften ergibt, daß die Autoren den Originaltext des Paracelsus nicht wörtlich übernahmen, sondern Veränderungen zu ihrer Präzisierung und Vereinfachung vornahmen. Die Texte beginnen mit der formalen Rezeptverschreibung, wie sie in den individuellen ärztlichen Verordnungen auch heute noch üblich ist. Dem Zeichen $R_{x}$ (Recipe!) folgt die Angabe der Bestandteile in lateinischer Sprache im Genitiv; oft werden auch alchimistische Zeichen verwendet. Immer sind Myrrha, Aloe und Crocus vorgeschrieben. Die zu verwendenden Drogen werden dann exakter umschrieben (Myrrha Alexandrina, Aloes Epatica und Crocus Orientalisseu Ungaricus). Scheuchzer ${ }^{3}$ läßt Terra Fol. Tartari anstelle von Alkali Tartari und Sal Nitrum zusetzen. Die Gewichte der Ausgangsdrogen werden exakter; von der Bezeichnung ana (ana partes) I vierling, also von der Angabe in alten Gewichtsteilen, wird übergegangen zum Nürnberger Apothekergewicht, das 1555 von der Reichsstadt Nürnberg eingeführt und auch bei uns bis zum Jahre 1856, der Einführung des schweizerischen Medizinalgewichtes, gebraucht wurde. Die Vorschrift von Croll ${ }^{17}$ läßt von jeder Droge 4 Unzen $(=4 \times 29,8 \mathrm{~g})$ verarbeiten. Die Herstellungsvorschrift nach Paracelsus ${ }^{16}$ weist noch deutlich alchemistische Züge auf; die folgenden sind schon präziser und leichter verständlich. Croll ${ }^{16}$ läßt die Drogen fein pulverisieren, dann mit Spiritus Vini befeuchten und fügt Oleum Sulphuris hinzu. Hierauf wird zwei Monate lang der Digestio und Circulatio (eine Art Kochen am Rückflußkühler) unterworfen; die überstehende Tinktur wird abgezogen, der Rückstand mit Spiritus Vini zwei Monate ausgekocht, die Flüssigkeit abgetrennt und vom Bodensatz abdestilliert. Die Auszüge und das Destillat werden vereinigt und ergeben das «Elixir Proprietatis», nachdem die Auszüge einen weitern Monat erhitzt wurden. Scheuchzer ${ }^{3}$ weist auf die Möglichkeit der Herstellung mit Spiritus Sulphuris oder Sal comune Tartari hin. Die Zubereitung dieses Elixirs ist somit sehr umständlich, schreibt es doch das Pulverisieren, Befeuchten, Digerieren und Kochen, sowie eine Destillation vor. Die Hersteller stellten sich ohne Zweifel vor, es würden die Bestandteile der Drogen nicht nur extrahiert, sondern während der langen Digerier- und Kochdauer untereinander und mit dem Schwefelöl 


\section{ELIXIR PROPRIETATIS}

\section{PARACELSL}

Be. Myrrhir Alexand.

Aloës Epat.

CrociOricntalis ana unc. 4 .

Pulverifcntur urnia diligcnecr, $\&$ in vitrum indaneur, humcetando optimo $S_{p}$ ritu vini Alcoholifato, poftea affunditur reEt ficatum olcum Sulphuris per campana: factum (praparcturillud olcum è Sulphurc flavo vel fubgiyfco, tcmpore pluvio. ,f majorem copiam habebis) ad cminentiain 4 . digitorum, ponatur in digctione $\&$ cis culationeper duos menfes, tandem quod tinetum \& cxtractum fuit perinclinatior fepara,materix in fundoreftanti bonum Spiritum vini affunde, \& circulando per ines fes duos itcrum coloratum cxtrahe, fcpara, ac prioriliquori solorato mifcc. Fxces fundolentc̀ deftillcntur, quod primum cgrcditur, addatur fupcriori Tinctur $x, \& i$ permenfen adhuc fine deftillatione circulcntur. Sapor cft fuavis, non amarus, mu to minus Empyrcuma fapicns, ut ficrifolct vulgariter deftillando.

Diligentcr obfervandum, materiam primo irrorandamSpiritu vini , tie fiat qu: if pafta, \& demum olcum Sulphuris affundendum, alioquin tota matersa in nigred. nem comburctur, id quod Paracelfus ftudiosc̀ occultayit:

\section{Vires \& UIfus.}

Balfamum prifcorum cht, ut Paracclfus ait, tenuisum prorfus partium calcfacien \& ficcans, ficque à putrefiactione confcrvans. Elt ņcaffcetum Elixir, in eo fune omn virtutes. Naturalis-Balfami, \& virtus confervativa muxima, præcipuè in fenibus.

1. Mirandainaffectionib. Thoracis \& Pulmonis pratat .

2. Adycrfus peftiferæ Luis contagia populatin graffaitia, ä̈remque corrupi mịirècflicax.

3. Humores Venericuli diverfos educit, ftomachum cum intcltinis confor cat. \& à doloribusprixforvat. ficat,

ه. Hecijcie, Catharrofis \&. Tuffentibus in genere fubvenit, \&e pectus mundi

5. Capitis \& Stomachi refrigerationiprodeft.

6. Homicraniam \& Vertigincm curat.

7: Oculorum caligincm depellit.

8. Cor \& memoriam confortat.

9. Paulatim Pruritum, dolorem item Iliorum \& Coftar um levat,

10. Calculos Rentum rumpit

II. Quartanam curat.

15. A Paralyli\& Podagraprexfervat.

I3. Intellectum \& omnes fenfus acuit.

14. Mclancholiamaufert, \&letitiamadfert. dat.

15. Senceturen fortiffima juventutis converfatione arcet, accanitiem retar-

16. Vitam ex ninvia cibi \& potus fumptione ąbbreviatam prolongat.

17. Vulnera \&ulcera interiora brevi conglutinat tempore.

18. 'Omnes demum tàm calidas, quàm frigidas infirmitates occulta quadàm pro. prictate curat.

\section{Dofis。}

à 6. ad 10: \& 12: guttàs in vinovécl aqua convenienti \& fpecifica:

Abb. 8. Vorschrift für das Elixir Proprietatis Paracelsi nach Oswald Croll in seiner «Basilica Chymica» 
Elioir Aroprietatis

justa Irdicripti inov:

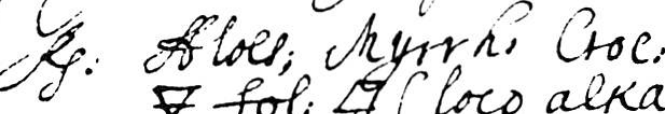

$\forall$ fol: 1 Coco alkalitit

Q)

pelilerum trium âa, un riuias dupli. kerant inotect quobi in thoct anio, king

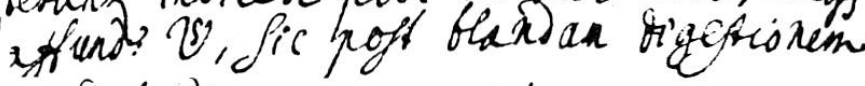

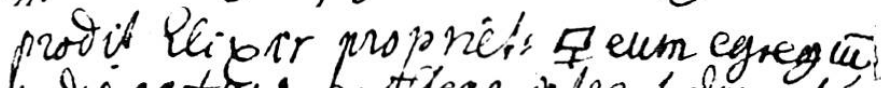

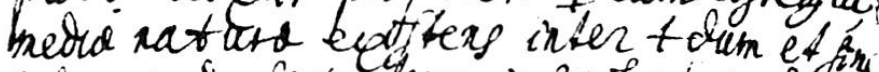

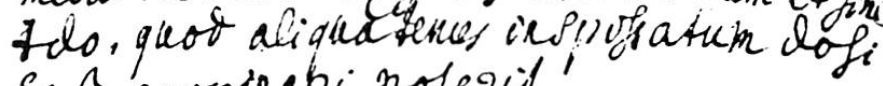
cos propinaki poteni.

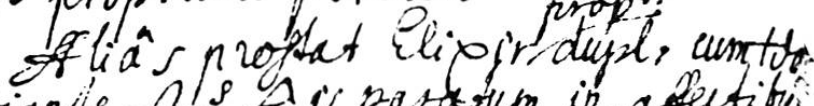
metiante $\Omega \cdot s$ sis parctum, in affection

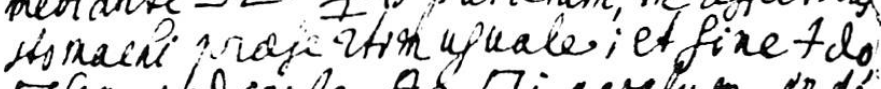

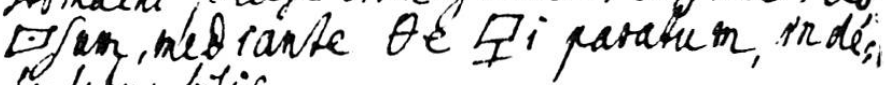
fuetibus bitis.

bejty Elix ir "rops" in corrigendo fo"

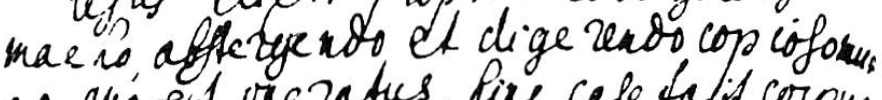
co, quo of vic zatus, line cale falis corpus

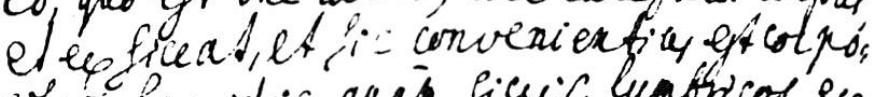

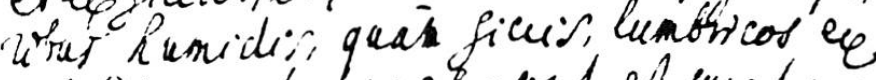
putregine octos prejer vat, et wert pro.

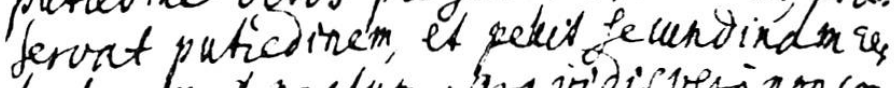

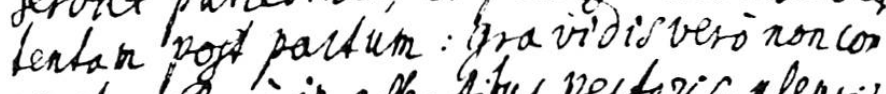
venit. Pons in affectitus pectoris, plearitis de et Rerix reumonio odppu rata, quonis.

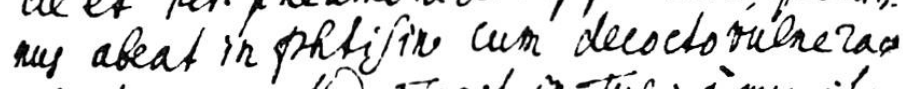

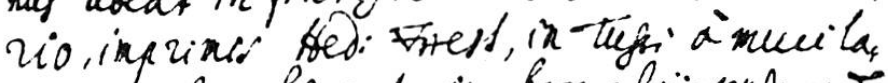
wine ingto lastate in bronchis pumonos. chartigine et tonbecilicitatevifus, octive

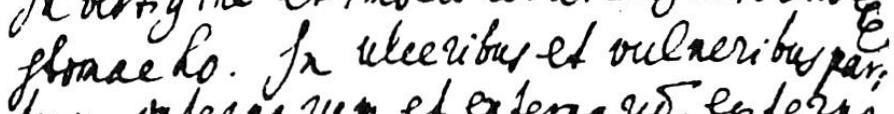

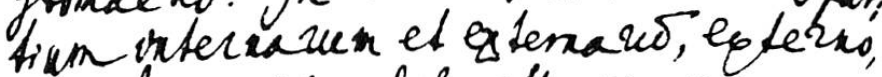

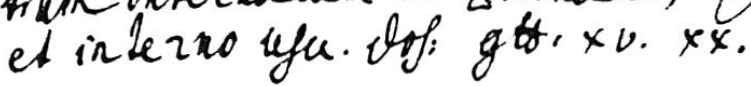

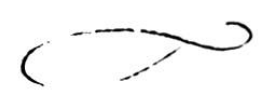

Abb.9. Text für das Elixir Proprietatis Paracelsi in Scheuchzers «Pharmacia Contracta» 
Reaktionen zu den für die Wirkung verantwortlichen Stoffen eingehen. Unverständlich lang ist auch die Herstellungszeit von insgesamt fünf Monaten! Dieses Beispiel steht nicht für sich allein, vielmehr sind einige der von Scheuchzer ${ }^{3}$ beschriebenen Composita kompliziert zusammengesetzt (Polypharmaka) und mit anspruchsvollen Apparaturen mühsam herzustellen. Allerdings zeichnet sich bei vielen Vorschriften, vor allem bei den Rezepten das Streben nach Vereinfachung ab.

Die genaue Beschreibung der Herstellung muß verstanden werden aus dem Bestreben, beim Mangel an Prüfungsvorschriften doch eine gewisse Normung der Endprodukte zu erreichen. Dieses Vorgehen beruht auf der Annahme, daß die genaue Einhaltung einer Herstellungsvorschrift zu stets gleich zusammengesetzten Präparaten von gleichartigen Eigenschaften führen müsse, was schon infolge der Inkonstanz der Ausgangsstoffe nicht möglich ist. Eine Prüfung der Arzneimittel mußte sich infolge des Fehlens von physikalischen und chemischen Methoden auf den Nachweis der Identität der Zubereitung aufgrund ihres Aussehens, Geruches und Geschmacks beschränken. Physikalische und chemische Identitäts- und Reinheitsprüfungen sowie Gehaltsbestimmungen fanden erst im 19. Jahrhundert Eingang.

Von Interesse ist es auch, die Angaben über die Wirksamkeit und die therapeutische Anwendung der obigen Elixir-Proprietatis-Vorschriften miteinander zu vergleichen. Mit den Elixieren soll Paracelsus nach Ernst Darmstädter ${ }^{19}$ bezweckt haben, Konservierungsmittel einzuführen, die den lebenden Körper gesund und frisch erhalten können. Ein solches Präparat sollte den Körper völlig und überall durchdringen und ihn vor «Fäulniserscheinungen» schützen. Folgen wir dem Text des Paracelsus (Abb. 7): «in dem ist alle tugend der natürlichen Balsamen und ist in ihm virtus conservativa etc....».

In der Tat enthält das «Elixir Proprietatis» mit Myrrhe und Aloe Stoffe, die fäulniswidrig sind.

Sehr einläßlich äußert sich Croll ${ }^{17}$ zu den Vires et Usus (siehe Abb. 8), indem er Bezug nimmt auf Paracelsus und die konservativen Eigenschaften in 18 Punkten weitausholend umschreibt. Auch Scheuchzer ${ }^{3}$ lobt die vorzüglichen Wirkungen. Damit wird das Elixier zum «unfehlbaren» Allesheilmittel.

Die Dosierung wird ebenfalls unterschiedlich angegeben. Croll ${ }^{17} \mathrm{emp}-$ fiehlt 6-10 und 12 Tropfen in Wein oder Wasser, während Scheuchzer ${ }^{3}$ 15-20, also die doppelte Menge vorschreibt. 


\section{Die Beurteilung der «Pharmacia Contracta» von J. J. Scheuchzer}

Die kritische Durchsicht dieses handschriftlichen Arzneiverordnungsbuches zeigt, daß es nicht allein eine gekürzte Fassung von Ludovicis «De pharmacia moderno saeculo accommodata» ist, sondern die einläßliche Beschreibung einer Auswahl seiner Arzneistoffe und Arzneipräparate. Die Gewinnung und Aufbereitung der Simplicia und besonders ihre Verarbeitung zu Arzneidrogenpräparaten (Composita) werden einläßlich und mit guter Fachkenntnis dargestellt. Dabei fällt besonders auf, daß sich der Arzt Scheuchzer sehr einläßlich mit Fragen der Arzneizubereitung befaßte. Er verfügte über gute Kenntnisse und bediente sich häufig der wichtigsten Quellen seiner Zeit. Dabei bezieht er sich aber nirgends auf die Arbeiten der Zürcher Autoren Gessner, Wolf, Ziegler und von Muralt.

Der behandelte Arzneischatz stand auf der Höhe der Zeit; nur die neuen ausländischen pflanzlichen Arzneidrogen aus Ostasien und Amerika fanden kaum Verwendung. Als Botaniker seiner Heimat hat Scheuchzer die inländischen Pflanzen bevorzugt. Die tierischen Drogen nahm Scheuchzer noch stark in Gebrauch. Vor allem aber erweist er sich als Anhänger der Chemiatrie, denn die im 16. und 17.Jahrhundert neu in die Medizin eingeführten anorganischen Stoffe fanden eine starke Berücksichtigung. Mit seinem Werke schuf er sich ein wertvolles, auf der Höhe seiner Zeit stehendes Hilfsmittel für seine ärztliche Praxis und die Ausbildung der angehenden Mediziner.

\section{Literaturübersicht}

${ }^{1}$ Steiger Rudolf, Verzeichnis des wissenschaftlichen Nachlasses von Johann Jakob Scheuchzer (1672-1733). Vierteljahrschr. der Naturf. Gesellschaft Zürich 78 (1933) 45.

${ }^{2}$ Ludwig, Daniel, «De pharmacia moderno saeculo accommodata», Editio tertia, Lipsiae 1696: ZBZ. Rx 273; Editio secunda, Lipsiae 1685: Med. Hist. Institut Universität Zürich 7 L 946.

${ }^{3}$ Scheuchzer, Johann Jakob, «Pharmaciae Contractae praesertim Ludovicianae Clarior Ideae», 1700; Bibl. Pharm. Inst. ETH-Zürich.

${ }^{4}$ Paullini, Kristian Franz, «Heilsame Dreckapotheke, wie nemlich mit Kot und Urin fast alle, ja auch die schwersten Krankheiten glücklich curiert werden»; Frankfurt a. Main 1696.

${ }^{5}$ Muralt von, Johann, «Pharmacopoea Domestica» 1685; ZBZ. MS VII 115 \& DE 1579.

${ }^{6}$ Ludwig, Daniel, «Compendium Materiae Medicae» 1698.

7 Lavater, Hans Conrad, «Laborbuch» 1672; ZBZ. Hs. Abt. FA. Lav. 1628.

${ }^{8}$ Lavater, Hans Georg, «Collectanea Chymica-Pharmacentica» 1674; ZBZ. Hs. Abt. FA Lav. 1657. 
${ }^{9}$ Fischer, Hans, «Johann Jakob Scheuchzer, Naturforscher und Arzt», Neujahrsblatt 1973 der Naturforschenden Gesellschaft Zürich, Verlag Leemann, Zürich 1973.

${ }^{10}$ Schneider, Wolfgang, «Lexikon zur Arzneimittelgeschichte», Bd. II (1974).

${ }^{11}$ Gessner, Conrad, «Thesaurus Euonymi Philiatri, De Remediis Secretis», I. Bd. 1552, II. Bd. 15: ZBZ. AE 604 und Med. Hist. Inst. ZH. 6 G 392.

12 Wolf, Caspar, «Diodori Euchyontis Polychymica», 1567 Zürich; ZBZ. AW 744.

${ }^{13}$ Ziegler, Adrian, «Pharmacopoea Spayrica» 1616 Zürich, ZBZ. AX 740.

${ }^{14}$ Muralt von, Johann, «Anatomisches Collegium» Nürnberg 1687; ZBZ. VI. 189.

${ }^{15}$ Schneider, Wolfgang, «Geschichte der pharmazeutischen Chemie», Verlag Chemie; S.101, 123 und 148 (1972).

${ }^{16}$ Sudhoff, Karl, «Sämtliche Werke des Theophrastus von Hohenheim», I.Abt. 3.Band S. 185-194 (1930).

${ }^{17}$ Croll, Oswald, «Basilica Chymica» 1609 Frankfurt; Med. Hist. Inst. Universität ZH, 7 C 944.

${ }^{18}$ Schröder, Johann, «Pharmacopoea Medico-chymica» 1649 Lugduni; Bibl. Pharm. Institut ETH Zürich und Med. Hist. Institut, Universität Zürich 7 S 381.

${ }^{19}$ Darmstätter, Ernst, «Arznei \& Alchemie: Paracelsus Studien»; in Studien zur Geschichte der Medizin. Verlag Ambrosius Barth, Leipzig, S. 56, 1931.

\section{Summary}

Johann Jakob Scheuchzer (1672-1733), the famous physician and natural scientist of Zurich, was the author of a Pharmacopoea Contracta of 1700. The manuscript had disappeared for a long time, but recently it was found in a library of Zurich. It is a collection of medical prescriptions for the use of the author and his students. The pages are partly an extract, partly an extention of the book De pharmacia moderno saeculo accomodata by Danielis Ludovicus (Daniel Ludwig) in the edition of 1696. Scheuchzer's pharmacopoeia gives a good picture from the therapeutics and pharmaceutics at the end of the 17th century. He emploied above all medicinal herbs and only scarcely animal drugs; as a iatrochemist, he also prescribed very often chemical agents. Scheuchzer knew very much absout the making up of prescriptions. His pharmacopoeia was an up-to-date dispensary of that time.

Prof. Dr.sc.nat. Dr. med.h. c. Jakob Büchi

Alte Landstraße 98

CH-8700 Küsnacht 
